\title{
Participación corresponsable del estudiante en la evaluación. Una experiencia del Máster en profesorado.
}

\author{
José M. ${ }^{a}$ Falcó Boudet ${ }^{\mathrm{a}}$
}

${ }^{a}$ Dpto. Informática e Ingeniería de Sistemas (Facultad de Educación, Universidad de Zaragoza) chema.falco@unizar.es

\begin{abstract}
In this paper we analyze the effectiveness of an innovative approach to the evaluation in a teacher's training course which consists in making students jointly responsible for the process from its first phase: the definition of the assessment criteria. It began with an individual analysis and then a discussion among the group where students and teachers agreed on the criteria with which two of the tasks of the subject would be assessed. Later the students, using the established criteria, also assessed one of the activities of their peers. After the experience, it was evaluated both the subjective impact in relation to personal and group involvement in their learning and the impact on learning itself. The results have shown that student responsibility in their evaluation process results in an improvement of both.
\end{abstract}

Keywords: Co-assessment, Students involvement, Teacher training

\begin{abstract}
Resumen
En este artículo analizamos la eficacia de una experiencia de innovación en la evaluación en el ámbito del Máster en profesorado consistente en hacer a los estudiantes corresponsables del proceso desde la primera fase: la definición de los criterios. A partir de un análisis individual y tras un debate entre todo el grupo, alumnos y profesores consensuaron los criterios con los que se evaluarían dos de las tareas de la asignatura. Posteriormente los estudiantes, haciendo uso de los criterios que habían definido, también evaluaron una de las actividades de sus compañeros. Para evaluar la experiencia se valoró tanto el impacto subjetivo en relación a la implicación personal y grupal en su aprendizaje como su repercusión en el mismo. Los resultados han demostrado que la corresponsabilidad del estudiante en su proceso de evaluación se traduce en una mejora de ambos factores.
\end{abstract}

Palabras clave: coevaluación, participación de los estudiantes, máster en profesorado.

\section{Introducción}

El cambio de los sistemas educativos de cualquier nivel y tipo de formación a un paradigma centrado en el alumno requiere también de un cambio en consecuencia del modelo de 
evaluación. Sanmartí (2007) considera hay que implicar a los estudiantes tanto en el proceso de su propia evaluación como en la de sus compañeros, con criterios y conocimiento. Son cada vez más los profesores que requieren la participación de sus alumnos en ella. Hounsell y otros (1996) clasificaron en tres las distintas maneras en las que esto se lleva a cabo: la autoevaluación, la evaluación entre iguales, que incluye tanto la calificación de trabajos como el proporcionar feedback, y el acuerdo con los profesores de alguno de los aspectos del proceso. Biggs (2005) apunta que los estudiantes también deberían participar en la primera fase del mismo: la definición de los criterios de evaluación. Lyons (1989 citado por Dancer y Kamvounias, 2005) defiende que la participación de los estudiantes en el establecimiento de los criterios de evaluación es en sí misma una experiencia de aprendizaje porque requiere que los alumnos reflexionen sobre lo que está "bien hecho" y "mal hecho" al realizar una tarea.

Los estudiantes del Máster Universitario en Profesorado de Educación Secundaria Obligatoria, Bachillerato, Formación Profesional y Enseñanzas de Idiomas, Artísticas y Deportivas de la Universidad de Zaragoza deben desarrollar, entre otras, la capacidad juzgar el aprendizaje de sus futuros alumnos. Para ello durante el curso 2014-15 se propuso a los estudiantes de la asignatura de Diseño, organización y desarrollo de actividades para el aprendizaje de Tecnología e Informática una experiencia en la que debían ser jueces de su propia práctica y de la de sus compañeros y así tomar conciencia de lo que habían aprendido y de lo que necesitaban aprender, sin delegar este papel únicamente en el profesor.

En cursos anteriores los profesores de esta asignatura ya habían propuesto a los alumnos la realización de actividades de evaluación entre iguales (Falcó, 2015), colaborando en la calificación final, siguiendo a Hamodi y López (2012) que han llegado a la conclusión de que los estudiantes prefieren la evaluación colaborativa con el profesor antes que la evaluación entre iguales. En esta ocasión se amplió la participación a la primera fase el proceso de evaluación para que desde su inicio los alumnos fueran corresponsables del mismo, fijando de acuerdo con los profesores los items con los que se evaluaría el resultado de su proceso de aprendizaje.

\section{Objetivos}

Esta experiencia tiene dos objetivos generales, uno en cuanto a los aprendizajes de la asignatura y otro en cuanto al desarrollo de habilidades profesionales que los estudiantes esperan.

Los profesores de la asignatura buscaban que mediante la coevaluación los estudiantes potenciaran el aprendizaje de los compañeros y el propio. Hacer al estudiante no solo conocedor sino también responsable de establecer qué debe aprender debería repercutir en una mejor comprensión de los contenidos de la asignatura y en la mejora de sus aprendizajes.

Además, participar en el proceso de evaluación les proporciona formación necesaria en el ejercicio de su profesión en cuatro aspectos:

2016, Universitat Politècnica de València

Congreso IN-RED (2016) 
- Conocieron el proceso de evaluación desde su inicio, incluyendo las acciones previas al hecho en si de evaluar: fijar los criterios, redactarlos de manera clara y sencilla y darlos a conocer al alumnado.

- Desarrollaron la competencia digital, mediante la práctica, haciendo uso de herramientas 2.0 como herramientas de evaluación.

- Conocieron mediante experimentación directa la utilización de diferentes mecanismos para reasignar responsabilidades en la evaluación implicando al alumno en su aprendizaje.

- Adquirieron sensibilización ante las posibles correcciones de la tarea de un docente por parte de otros miembros del equipo (claustro o departamento).

\section{Desarrollo de la innovación}

Como ha quedado indicado, esta experiencia se llevó a cabo durante el curso 2014-15 con los alumnos de la asignatura de Diseño, organización y desarrollo de actividades para el aprendizaje de Tecnología e Informática del Máster en profesorado de la Universidad de Zaragoza.

\subsection{Actividades}

La tarea principal de esta asignatura es el diseño de un proyecto de aprendizaje según la metodología del Aprendizaje Basado en Proyectos (ABP). Este proyecto se presenta ante todo el grupo de estudiantes "impartiendo una clase" de una de las fases del mismo.

La participación de los estudiantes en la evaluación del proyecto se llevó a cabo de la siguiente manera:

- Análisis individual de los aspectos que debe incluir un buen proyecto de aprendizaje.

- Análisis individual de los aspectos que debe tener en cuenta un profesor de secundaria en el aula para propiciar el aprendizaje de sus alumnos.

- Elaboración por consenso de los criterios de evaluación de los proyectos de aprendizaje y de la acción docente del profesor durante la clase. Se hizo en tres fases, proporcionándoles en cada una los "instrumentos y técnicas adecuados, que faciliten su participación en la evaluación” (Gil y Padilla, 2009).

Primero, tras el análisis inicial, los estudiantes divididos en grupos de 2 o 3 pusieron en común qué criterios les parecían pertinentes para evaluar su proyecto y para evaluar su acción como profesores y cuáles no.

Después hubo una puesta en común utilizando un documento de Google Drive compartido con toda la clase. A continuación, se debatieron cada uno de los items propuestos en el documento, uniendo algunos, matizando otros para que 
recogieran aspectos comunes a varios y eliminando los que no se consideraron pertinentes.

En la última tarea de esta fase, utilizando un cuestionario de Google Forms enviado por el profesor, ponderaron individualmente cada criterio de cero a tres.

- Diseño, en equipo o individualmente, de un proyecto de aprendizaje.

- Cada equipo seleccionó una fase de su proyecto y "dio una clase" a sus compañeros que en ese momento tenían el rol de alumnos de secundaria realizando un proyecto de aprendizaje.

- Evaluación individual de la "clase" que habían recibido: Utilizando los criterios consensuados cada estudiante evaluó la actuación "como profesor" de cada uno de sus compañeros.

La media de la evaluación cuantitativa que los estudiantes hicieron de la puesta en escena de los otros compañeros promedió con la del profesor y representó el $40 \%$ de la puntuación final de la asignatura.

\subsection{Evaluación de la experiencia}

El diseño de la experiencia incluyó la valoración de la misma por alumnos y profesores. Los alumnos evaluaron el impacto subjetivo en relación al interés de las actividades propuestas y a la implicación personal y grupal en el proceso de enseñanza - aprendizaje mediante un cuestionario anónimo online. Los profesores evaluaron la calidad de los aprendizajes logrados por los alumnos.

\section{Resultados}

En el análisis y la selección de los criterios participaron 12 de los 21 estudiantes matriculados en la asignatura (57\%). El número de estudiantes que evaluó a otros compañeros varió entre un escaso 33,3\% (7 alumnos) y un 67\% (14 alumnos), según los que asistieron cada día a clase.

El cuestionario fue contestado por 18 estudiantes, lo que representa el $85,71 \%$ de los alumnos matriculados en la asignatura.

\subsection{Valoración hecha por los estudiantes}

El cuestionario pedía la valoración de tres aspectos: su opinión en relación a la reasignación de responsabilidades, su valoración del impacto que la participación en la evaluación ha tenido en su aprendizaje, y su percepción del nivel de implicación en la evaluación de la asignatura. 


\subsubsection{Valoración de la reasignación de responsabilidades}

La tarea de participar en la elaboración de los criterios de evaluación obtuvo una valoración media de 3,78, siendo cero "Lo veo innecesario" y cinco "Me ha ayudado a saber qué es importante aprender en la asignatura".

La actividad de la coevaluación ha obtenido una valoración media de 4.

En ambos casos la puntuación mínima fue " 2 = Me ha ayudado poco". El detalle de estas valoraciones se puede ver en la tabla 1.

Tabla 1. Valoración de la reasignación de responsabilidades

\begin{tabular}{ccccccc}
\hline & $\mathbf{0}$ & $\mathbf{1}$ & $\mathbf{2}$ & $\mathbf{3}$ & $\mathbf{4}$ & $\mathbf{5}$ \\
\hline $\begin{array}{c}\text { Participar en la } \\
\text { elaboración de criterios }\end{array}$ & 0 & 0 & $16,67 \%$ & $16,67 \%$ & $38,89 \%$ & $27,78 \%$ \\
\begin{tabular}{c} 
Coevaluar las "clases" \\
\hline
\end{tabular} & 0 & 0 & $5,56 \%$ & $27,78 \%$ & $27,78 \%$ & $38,89 \%$ \\
\hline
\end{tabular}

\subsubsection{Valoración del impacto en el aprendizaje}

La percepción subjetiva de haber aprendido a diseñar un proyecto de aprendizaje mediante la participación en la evaluación ha obtenido una valoración media 3,88 (0: En absoluto; 5: Con total seguridad), mientras que la percepción de haber aprendido diseñar criterios de evaluación ha sido 3,82 .

\subsubsection{Percepción del nivel de implicación}

Llama la atención que, en media, consideran que el nivel de participación personal es superior al del grupo de clase, que a su vez es percibido como inferior al requerido por los profesores. En una escala de cero (participación inexistente) a cinco (participación muy

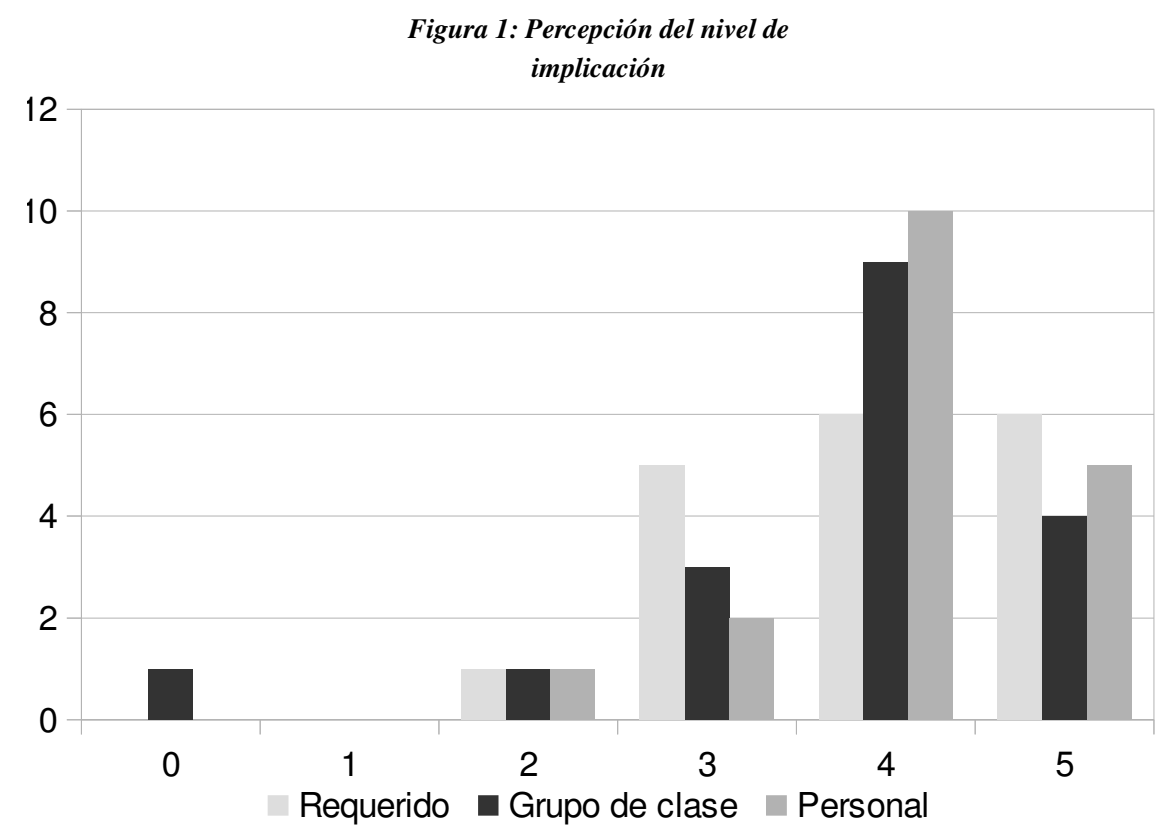

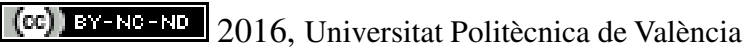

Congreso In-Red (2016) 
alta), la percepción del nivel de participación requerido es 3,94, mientras que la percepción del nivel de implicación del grupo es 3,72 y la percepción del nivel de implicación personal es 4,06. En el gráfico 1 se muestra un resumen comparado de las respuestas a estas tres cuestiones.

\subsection{Valoración hecha por el profesorado}

\subsubsection{Análisis cuantitativo}

Hemos confirmado la argumentación de Lyon (1989) de que haber analizado los criterios de evaluación y participar de manera activa para establecer cuales serán aquellos con los que se valoraría su proyecto repercutió en el aprendizaje, haciendo a los estudiantes ser conscientes de qué era importante tener en cuenta y qué no en el diseño del mismo. Prueba de ello es que la nota mínima $(4,8)$ la obtuvo un alumno que no asistió ningún día a clase y que no participó en el análisis y diseño de los criterios de evaluación. La calificación media de los otros seis proyectos es de 8,01. La calificación media en conjunto fue 7,61. Este mismo alumno fue el que obtuvo la calificación mínima de las otorgadas por los compañeros $(6,5)$ al "dar la clase". La calificación media de todo el grupo fue 8,65.

\subsubsection{Análisis cualitativo}

Los profesores de la asignatura analizaron comparativamente los criterios que propusieron los alumnos con los que utilizaban para evaluar los proyectos y presentaciones en cursos anteriores. El resultado es que lo esencial para valorar si los estudiantes habían aprendido a diseñar actividades de aprendizaje y a desarrollarlas en el aula está recogido en los criterios propuestos por el grupo. La comparación de ambas series de criterios está recogida en las tablas 2 y 3 .

Tabla 2. Comparación de los criterios anteriores con los propuestos por los alumnos para evaluar los proyectos de aprendizaje

\begin{tabular}{ll}
\hline \multicolumn{1}{c}{ Criterios anteriores } & \multicolumn{1}{c}{ Criterios propuestos por los alumnos } \\
\hline Maquetación y formato & \\
$\begin{array}{l}\text { Es realizable con los medios y tiempo } \\
\text { disponibles con un grupo de alumnos }\end{array}$ & Es aplicable a la edad de los estudiantes \\
Innovador & \\
Interdisciplinar y transversal & \\
$\begin{array}{l}\text { Conecta con los intereses de los alumnos y está } \\
\text { contextualizado }\end{array}$ & $\begin{array}{l}\text { Trata sobre un tema actual y/o de interés que } \\
\text { puede interesar a los alumnos }\end{array}$ \\
Incluye algún instrumento de autoevaluación & $\begin{array}{l}\text { Posibilita la realimentación para mejora } \\
\text { continua }\end{array}$ \\
Deja margen a la creatividad del alumno & $\begin{array}{l}\text { Fomenta la creatividad y el espíritu crítico de } \\
\text { los alumnos }\end{array}$
\end{tabular}




\section{Criterios anteriores}

Incluye mecanismos de atención a la diversidad

Detalla de manera clara los objetivos de aprendizaje

Fomenta el trabajo colaborativo

\section{Criterios propuestos por los alumnos}

Contempla atención a la diversidad

Define con precisión los criterios e instrumentos de evaluación de una unidad didáctica

Utiliza una metodología activa.

Plantea el aprendizaje de competencias básicas

Define de manera completa las fases del proyecto. Organiza y estructura el proyecto de manera clara con una adecuada temporalización.

Cita las fuentes bibliográficas siguiendo un formato estándar

Tabla 3. Comparación de los criterios anteriores con los propuestos por los alumnos para evaluar la clase impartida por los compañeros

\begin{tabular}{ll}
\hline \multicolumn{1}{c}{ Criterios anteriores } & \multicolumn{1}{c}{ Criterios propuestos por los alumnos } \\
\hline $\begin{array}{l}\text { Presenta la información de manera lógica y } \\
\text { ordenada. }\end{array}$ & $\begin{array}{l}\text { Concreción, claridad, amenidad y fluidez en la } \\
\text { presentación }\end{array}$ \\
$\begin{array}{l}\text { Fomenta la participación activa de los alumnos } \\
\text { en su propio proceso de aprendizaje. }\end{array}$ & $\begin{array}{l}\text { Consigue la atención y la motivación de los } \\
\text { alumnos }\end{array}$ \\
$\begin{array}{l}\text { Participan activamente todos los miembros del } \\
\text { equipo "docente". }\end{array}$ & $\begin{array}{l}\text { Los profesores interaccionan con la clase } \\
\text { (alumnos) }\end{array}$ \\
$\begin{array}{l}\text { El mensaje es claro y entendible. Los alumnos } \\
\text { entienden rápidamente la propuesta. }\end{array}$ & $\begin{array}{l}\text { Uso de un lenguaje comprensible por parte del } \\
\text { alumno }\end{array}$ \\
Utiliza herramientas TICS. & $\begin{array}{l}\text { Los medios y recursos utilizados son los } \\
\text { adecuados }\end{array}$ \\
& Originalidad en la idea de la presentación \\
\hline
\end{tabular}

$\overline{\text { Si bien los alumnos no dieron importancia al formato, probablemente porque es algo que ya }}$ saben hacer (todos los proyectos estuvieron bien presentados y maquetados) valoraron la necesidad de citar adecuadamente las fuentes. Donde los profesores de la asignatura pedíamos que "dejaran margen a la creatividad" los estudiantes piden que no solo se de margen sino que se fomente, añadiendo también el espíritu crítico. Si los profesores requeríamos que los proyectos fomentaran el trabajo colaborativo los estudiantes requieren que toda la metodología en conjunto sea activa. Si bien el aprendizaje colaborativo aporta capacidades específicas al aprendizaje que no aportan otras metodologías activas es cierto que en la esencia del ABP está el que sea un proceso activo. La propuesta de los estudiantes amplia el criterio referente a los objetivos de aprendizaje, pidiendo que no solo los criterios

\section{(cc) BY-NC-ND 2016, Universitat Politècnica de València}

Congreso In-Red (2016) 
sino también los medios de evaluación sean definidos con precisión. Definición que extienden a las fases y estructura del proyecto. En cambio, no dieron valor a la innovación, que trabajan en otra asignatura del Máster en profesorado, ni a la transversalidad que si bien no es un requisito imprescindible del $\mathrm{ABP}$ si que es recomendable en su diseño.

En el análisis comparativo de los criterios para valorar la clase impartida por los compañeros al presentar su proyecto se aprecia que los estudiantes recogen todos los criterios que valoraban los profesores en cursos anteriores y que los amplían dando importancia a la originalidad. También es reseñable que las TIC no aparecen mencionadas explícitamente y que en cambio valoran que los medios sean los adecuados. Hay otra diferencia importante en cuanto a la participación de los alumnos. Mientras los profesores de la asignatura consideran que se debe mantener activa la participación de los alumnos durante todo el proceso los estudiantes solo han considerado que deben captar la atención, dado que su visión de conjunto es más reducida: ellos se preocupan de "la clase" que tienen que impartir mientras que los profesores valoran el desarrollo de toda la actividad de aprendizaje, independientemente del número de sesiones que dure.

\section{Conclusiones}

Esta experiencia supuso un modelo de evaluación innovador en una doble vertiente. Por un lado requería la participación responsable del estudiante para establecer los criterios de evaluación de la asignatura. Por otro requería un análisis crítico para coevaluar responsablemente las actividades de sus compañeros.

Los resultados muestran que se alcanzaron los objetivos establecidos al inicio del proyecto, habiendo conseguido que los alumnos del Máster en profesorado diseñaran buenos proyectos de aprendizaje y que se implicaran y responsabilizaran en la evaluación de sus aprendizajes y los de sus compañeros.

La participación de los estudiantes en el diseño de los criterios de evaluación propició que reflexionaran sobre aquellos elementos más importantes a tener en cuenta al diseñar una actividad de aprendizaje, dando como resultado la propuesta de unos criterios similares en lo esencial a los utilizados por los profesores de la asignatura en cursos anteriores.

La coevaluación, esto es, la evaluación de la acción docente de otros compañeros en paralelo a la evaluación de los profesores, desarrolló la capacidad de análisis crítico en relación a cómo un docente crea (o destruye) entornos de aprendizaje.

La percepción global de los estudiantes es que se sintieron parte importante en la evaluación de los aprendizajes.

La experiencia aquí descrita se ha circunscrito a una asignatura de una especialidad específica del Máster en profesorado pero, apoyándonos en los resultados positivos obtenidos, durante el curso 2015-16 la estamos desarrollando en otra asignatura de la especialidad. Además, opinamos que es transferible a otras titulaciones y grados universitarios. La participación de los alumnos en el diseño y concreción de los criterios de evaluación de una de las actividades y la posterior evaluación de la misma realizada por 
otros compañeros mejoran implicación de los estudiantes en la asignatura y son valoradas por ellos como una ayuda para mejorar los aprendizajes.

\section{Referencias}

BiggS, J.B. (2005). Calidad del aprendizaje universitario. Madrid. Nancea

DANCER, D., y Kamvounias, P. (2005). "Student involvement in assessment: a project designed to assess class participation fairly and reliably". Assessment \& Evaluation in Higher Education, v 30 n 4, 445-454.

FALCó, J.M. (2015). "El portafolio digital como instrumento de evaluación formativa en el Máster en Profesorado" en Alejandre, J.L. Buenas prácticas en la docencia universitaria con apoyo de TIC. Experiencias en 2015. Zaragoza: Prensas de la Universidad de Zaragoza. 125-134.

GiL, J. y Padilla, M. T.. (2009). "La participación del alumnado universitario en la evaluación del aprendizaje”. Educación XX1, v 12 n 1, 43-65.

HAMOd, C. y LoPEZ, A. T. (2012). "La evaluación formativa y compartida en la Formación Inicial del Profesorado desde la perspectiva del alumnado y de los egresados". Psychology, Society, \& Education, v 4 n 1, 99-112.

Hounsell, D., McCulloch, M., y ScotT, M. (1996). "The ASSHE Inventory: Changing Assessment Practices in Scottish Higher Education”. Universities' and Colleges' Staff Development Agency, Ingram House, 65 Wilkinson St., University of Sheffield, Sheffield, Scotland S10 2GJ, United Kingdom.

SANMARTí, N. (2007). 10 ideas clave. Evaluar para aprender. Barcelona: Grao. 